\title{
Assessment of skin response in T4b breast carcinoma patients post-neoadjuvant chemotherapy
}

\author{
Abhishek Sharma ${ }^{1}$, Shagun Mahajan ${ }^{1}$, Sanjit Kumar Agrawal ${ }^{1}$, Rosina Ahmed $^{1}$ and Debdeep Dey ${ }^{2}$ \\ ${ }^{1}$ Department of Breast Oncosurgery, Tata Medical Center, Kolkata 700156, India \\ ${ }^{2}$ Department of Pathology, Tata Medical Center, Kolkata 700156, India
}

\section{Abstract}

Background: Breast cancer patients with skin ulcerations, satellite nodules or Peau d'orange at presentation are classified with stage 4 breast cancer (T4b). Neoadjuvant chemotherapy (NACT), followed by mastectomy, is the commonly accepted treatment in such patients for fear of adverse outcomes with breast conservation surgery (BCS) and uncertainty over sparing initially involved skin irrespective of the response to chemotherapy. Identifying patients with skin resolution post-NACT can help surgeons in decision-making.

Aim: To assess skin response in T4b breast cancer patients post-NACT and find the correlation between various clinical and pathological factors associated with no skin involvement on final histology.

Methodology: Records of breast cancer patients managed at the Tata Medical Center, Kolkata, with NACT for T4b breast carcinoma patients who underwent mastectomy were reviewed between January 2014 and December 2018. Final histology was checked for dermal involvement with the tumour. The Mann-Whitney $U$ test was used for continuous variables for descriptive data, and Pearson's chi-squared and Fischer's exact tests were applied for categorical data. $p$-value $<0.05$ was taken as significant.

Results: A total of 285 records mentioning skin involvement were reviewed, out of which 111 patients fulfilled the AJCC criterion. The median age at diagnosis of T4b breast cancer was 50 years. The median clinical size pre-chemotherapy was $7 \mathrm{~cm}$. Residual median tumour size on final histology was reported as $1 \mathrm{~cm}$. 78/111 patients showed a postNACT response of $50 \%$ or more, and $43 / 111$ showed a response of more than $90 \%$. $57(51.4 \%)$ patients showed skin involvement on final histopathology, while 54 (48.6\%) patients did not.

ER negative tumours were more likely to show no dermal involvement $(p=0.006)$. Residual tumour size of less than $1 \mathrm{~cm}$ on final histology $(p<0.05)$ and nodal stage were significant predictors of dermal response.

Conclusion: Approximately half of the T4b breast cancer patients showed resolution of dermal skin involvement post-NACT. ER negative and those with residual tumour size

Correspondence to: Abhishek Sharma Email: abhishek.sharma@tmckolkata.com

ecancer 2021, 15:1271

https://doi.org/10.3332/ecancer.2021.1271

Published: $28 / 07 / 2021$

Received: 11/04/2021

Publication costs for this article were supported by ecancer (UK Charity number 1176307).

Copyright: (c) the authors; licensee ecancermedicalscience. This is an Open Access article distributed under the terms of the Creative Commons Attribution License (http:// creativecommons.org/licenses/by/4.0), which permits unrestricted use, distribution, and reproduction in any medium, provided the original work is properly cited. 
less than $1 \mathrm{~cm}$ post-NACT are more likely to show dermal resolution. This can help surgeons plan a BCS or skin sparing mastectomy for such patients who usually end up having a mastectomy.

Keywords: T4b, skin response, NACT, breast cancer

\section{Introduction}

Breast cancer is the most common cancer among women. Approximately 2.1 million new cases were estimated to occur worldwide in 2018 [1]. Breast cancer lesions with oedema (including Peau d'orange), satellite nodules confined to the same breast and ulceration of skin are classified as T4b, which is essentially a clinical classification [2].

Very few studies of patients with locally advanced breast cancer (LABC) who underwent neoadjuvant chemotherapy (NACT) and conservative surgery exist, but they have shown breast-conserving surgery as a safe and effective therapy for carefully selected patients [3, 4].

In developing countries like India, a big chunk of the patients present with LABC, many of whom also have skin involvement or the tumour is quite close to the skin [5]. The management of these lesions is controversial. Traditionally, they have been placed in the highest T subgroup (T4), but many researchers have questioned this approach.

T4b breast cancer is poorly described in terms of its biological behaviour or its response to NACT. Furthermore, most of the few attempts to study T4 disease tend to club inflammatory breast cancer skin changes with non-inflammatory breast cancer, even though the two are different clinical entities [6-10].

T4b lesions undergo NACT, but an overwhelming majority of patients who present with clinical T4b are still treated with a mastectomy after NACT because it is 'assumed' that the local failure rates with breast preservation would be unacceptably high [6]. But this may not be true. Multiple studies have shown that breast conservation surgery (BCS) is safe and feasible in appropriately selected T4b patients. However, even if surgeons attempt BCS, there is an inclination to include the initially involved skin in the BCS specimen, thereby reducing the aesthetic outcome of BCS. This decision is sometimes taken without considering the post-NACT response.

To the best of our knowledge, there is a paucity of research to assess the skin response after NACT in T4b. It is, however, an important question. Does the tumour regress from the skin after NACT and are there any predictors of response? The answer to this question will help surgeons make informed decisions when choosing to do BCS or skin sparing mastectomy (SSM) in appropriately selected T4b breast cancer patients.

\section{Methodology}

This was a single institute retrospective cohort study. Data were retrieved from prospectively maintained institutional Redcap database and clinical records. The study was approved by the institutional ethics committee with waiver number EC/WV/TMC/46/20.

Inclusion criteria encompassed all patients fulfilling the American Joint Committee on Cancer (AJCC) criterion of T4b, namely satellite nodules on the ipsilateral side, ulceration, Peau d'orange and oedema as judged by the treating surgeon on the first visit and who subsequently underwent mastectomy post-NACT. Patients who received less than four cycles of anthracycline and/or taxane-based chemotherapy, those who had more than 12 weeks gap from the end of chemotherapy to surgery and those who progressed or developed metastatic breast cancer were excluded from the study. Patients clinically labelled as skin free, but had mammographic evidence of skin involvement, were also not included in this study in keeping with the AJCC criterion [2].

A total of 285 consecutive patients with documented clinical skin involvement between January 2014 and December 2018 were screened for study eligibility criteria. Interestingly, the AJCC classification was not always followed when labelling 'skin involved' in the clinical record. 
Of the 285 patients, only 111 fulfilled the AJCC criterion for T4b. We mention this to highlight that T4b disease is poorly understood or classified even in tertiary cancer centres.

The standard NC regimen consisted of four cycles of AC (Doxorubicin [Adriamycin] $60 \mathrm{mg} / \mathrm{m}^{2}+$ Cyclophosphamide $600 \mathrm{mg} / \mathrm{m}^{2}$ ), followed by 4 or 12 cycles of $T$ (Taxol ${ }^{\circledR}$ [paclitaxel] $175 \mathrm{mg} / \mathrm{m}^{2}$ or $80 \mathrm{mg} / \mathrm{m}^{2}$, respectively). Only few HER2-positive (7/42) patients received Trastuzumab in addition to AC-T.

All patients with T4b disease underwent mastectomy with axillary dissection within a median of 3 weeks after completion of NACT as per institutional protocol. The final histopathology report was noted for residual skin involvement. Dermis involved and/or dermal lymphovascular invasion was taken as the marker for residual skin involvement.

\section{Statistical analysis}

Data normalcy was checked by the Shapiro-Wilk test. For descriptive data, Mann-Whitney U test was used for continuous variable, and Pearson's chi-squared/Fisher's exact tests were applied for categorical data. Multivariate analysis was carried out by using logistic regression. $p$-value $<0.05$ was taken as significant. All analyses were carried out with the Statistical Package for the Social Sciences statistical software package, version 25.

\section{Results}

\section{Patient characteristics}

The median age at diagnosis of T4b breast cancer was 50 years. $62.2 \%$ of the patients were postmenopausal. Most patients underwent surgery within a month of completing chemotherapy. $85.6 \%$ of the patients received AC-T regimen and $6.3 \%$ patients received Trastuzumab along with anthracycline and taxanes. The median time between completion of NACT and surgery was 29 days.

\section{Tumour characteristics}

The median clinical sizes pre-chemotherapy were 7 and $3 \mathrm{~cm}$ post-chemo. The residual median tumour size on final histology was reported as $1.6 \mathrm{~cm} .64 .9 \%(n=72)$ of the patients had grade three tumour. The majority of the patients were oestrogen receptor-positive $(57.7 \%)$ and progesterone receptor-negative (54.1\%). 42 (37.8\%) patients were HER2 receptor-positive. Only 6.4\% (7/42) of HER2+ patients received anti-HER2 therapy.

Table 1a and b summarise the demographics of the study population.

Table 1(a). Demographics of the study population.

\begin{tabular}{|l|c|}
\hline \multicolumn{1}{|c|}{ Parameters } & Median (range) \\
\hline Age (years) & $50(23-73)$ \\
\hline Time between chemotherapy and surgery (Days) & $29(20-180)$ \\
\hline cT size before chemotherapy (cm) & $7(2-15)$ \\
\hline cT size post-chemotherapy $(\mathrm{cm})$ & $3(0-9)$ \\
\hline cT ratio before and after chemotherapy & $0.5(0-1)$ \\
\hline pT size $(\mathrm{cm})$ & $1.6(0-13.3)$ \\
\hline
\end{tabular}


Table 1(b). Demographics of the study population.

\begin{tabular}{|c|c|}
\hline Parameters $(n=111)$ & $n$ (percentage) \\
\hline \multicolumn{2}{|l|}{ Body mass index } \\
\hline Underweight & $5(4.5)$ \\
\hline Normal & $34(30.6)$ \\
\hline Overweight & $42(37.9)$ \\
\hline Obese & $30(27)$ \\
\hline \multicolumn{2}{|l|}{ Menopausal status } \\
\hline Premenopausal & $42(37.8)$ \\
\hline Postmenopausal & $69(62.2)$ \\
\hline \multicolumn{2}{|l|}{ NACT regimen } \\
\hline$A C-T$ & $95(85.6)$ \\
\hline$A C$ & $9(8.1)$ \\
\hline AC-TH & $7(6.3)$ \\
\hline \multicolumn{2}{|l|}{ Skin involvement on final histology } \\
\hline Involved & $57(51.4)$ \\
\hline Not involved & $54(48.6)$ \\
\hline \multicolumn{2}{|l|}{ Grade } \\
\hline 1 & $2(1.8)$ \\
\hline 2 & $37(33.3)$ \\
\hline 3 & $72(64.9)$ \\
\hline \multicolumn{2}{|l|}{ Oestrogen receptor status } \\
\hline Positive & $64(57.6)$ \\
\hline Negative & $47(42.4)$ \\
\hline \multicolumn{2}{|l|}{ Progesterone receptor status } \\
\hline Positive & $51(46)$ \\
\hline Negative & $60(54)$ \\
\hline \multicolumn{2}{|l|}{ HER2 receptor } \\
\hline Negative & $47(42.3)$ \\
\hline Equivocal & $22(19.8)$ \\
\hline Positive( without Trastuzumab) & $35(31.5)$ \\
\hline Positive ( with Trastuzumab) & $7(6.4)$ \\
\hline
\end{tabular}

\section{Variables of response}

Overall, 57 (51.4\%) patients showed skin involvement (dermal involvement) on final histopathology report and 54 (48.6\%) patients did not show skin involvement. Only 10 (9\%) patients showed a pathological complete response (PCR), but 19 patients (17\%) had complete response in breast. 78/111(70.27\%) patients showed a post-NACT response of 50\% or more regression in tumour size, while $43 / 111(38.73 \%)$ showed a response of $90 \%$. 
The dermal response had a significant association with hormone receptor positivity. Patients with ER-positive tumours were less likely to have dermal regressions (40/64) than ER-negative tumours $(26 / 47)(p=0.006)$. A similar association was also noted with PR negativity $(p=$ 0.001). The number of patients who were HER2+ and received anti-HER2+ therapy was too small and, therefore, not subjected to statistical analysis.

Table 2 Correlation between clinicopathological variables and skin (dermis) involvement. A significant $p$-value is seen with ER-negative tumours and PR-negative tumours. Residual tumour size on final histopathology and $\mathrm{N}$ stage also showed significant $p$-values.

The dermal response was independent of age, initial tumour size or grade of tumour. It was also independent of BMI or menopausal status. The size of the residual tumour on histology was also significant. Patients with $<1 \mathrm{~cm}$ tumour size on final histology were more likely to have dermal regression ( $p<0.001)$. Out of the 40 patients with residual size $<1 \mathrm{~cm}, 32(80 \%)$ patients had no dermal involvement, while out of the 71 patients who had $>1 \mathrm{~cm}$ residual tumour involvement, 49 (69\%) patients had dermal involvement $\left(\chi^{2}=24.6, p<0.01\right)$.

\section{Discussion}

Breast tumours having significant skin involvement have been classified as T4b since the first edition of the AJCC staging system, published in 1977 [7]. Management of T4b lesions is complex and challenging [8, 9]. Various authors have questioned placing these lesions into the highest T category only because of skin involvement [10,11]. Once a lesion is placed in the T4 category, it becomes 'unfit' for breast conservation. This is clearly visible in the bias shown towards these lesions by excluding them from most large studies on BCS [12-14] after NACT.

Silverman et al [11] have shown that T4b lesions have the same prognosis as their counterparts of identical size and nodal status if skin involvement was ignored. They have called for treating these lesions based on their size and biology and to not be alarmed by their T4 status. Guth et al [10] have called placing lesions with skin involvement into T4 category 'a historical mistake'. Their logic that skin involvement may merely be a coincidence of a smaller lesion which originated near to skin envelope certainly finds merit.

This brings us to question as to why these tumours usually get denied BCS or SSM after NACT. It is conveniently assumed that even if T4b lesions showed good clinical response to NACT, they should still undergo mastectomy because of high failure rates [9]. Large trials that have showed BCS efficacy after NACT in 'large operable tumours' used a cut-off of 3 or $5 \mathrm{~cm}$ tumours but excluded T4b lesions from their study population [12-14]. Hence, there is a paucity of data to decide for or against this logic. In fact, there is a lack of data to assess how noninflammatory skin involved tumours behave after NACT. It is now generally accepted in post-NACT patients that 'neomargins' during surgery should be based on residual tumour and not the original tumour. The same logic could be extrapolated to skin response.

Our data show that in T4b breast cancer, the PCR was 17\% in the breast and overall PCR (breast + axilla) was $9 \%$. It is in accordance with similar findings from other studies around the world. Shen et al ([6)] reported a PCR of $24 \%$ in a selected group of T4b patients. Mclntosh et al [14] from Aberdeen breast clinic demonstrated a 17\% complete clinical response in a subset of T4b breast cancers [14]. We also report that $78 / 111$ (70.27\%) patients showed a post-NACT response of 50\% or more and $43 / 111(38.73 \%$ ) showed a response of $90 \%$ or more. This is despite the fact only 7/42 patients received Trastuzumab in our study, mainly due to financial constraints. If Trastuzumab were added to the chemotherapy, the above figures are expected to improve. All these patients are candidates for BCS or SSM and can avoid mastectomy.

Our study also visits the perennial question of what is a T4b tumour. Historically, any skin change has been considered T4b, and clinicians have been guilty of not adhering to AJCC classification. In our study, only 111 out of 285 (38\%) patients initially classified as skin involved actually fulfilled the AJCC criterion. Many of those not fulfilling the AJCC criterion had residual dermal involvement on the final histopathological examination (HPE). Other studies have also described this problem. In an international survey, 70\% of clinicians wrongly identified the criteria for T4b disease, leading the authors to caution the interpretation of data (1990-2000) of the T4b subset [15]. In fact, it was only the 2001 supplement of Tumour Node Metastasis (TNM) classification that clarified that the histological involvement in the absence of classical clinical signs does not constitute T4b [16].

The median tumour size of T4b tumours in our series was $7 \mathrm{~cm}$ (range $=2-15 \mathrm{~cm}$ ). Some of the other series have reported a similar median tumour size $[6,17,19]$. The dermal response was independent of initial tumour size, grade or menopausal status. It was also not dependent on BMI, duration of chemotherapy or multifocality of the tumour. 
Table 2. Demographics of the study population (continued).

\begin{tabular}{|c|c|c|c|c|c|}
\hline Variable & Total $(n=111)$ & $\begin{array}{c}\text { Skin (dermis) not } \\
\text { involved on final } \\
\text { histology } n(\%)\end{array}$ & $\begin{array}{c}\text { Skin (dermis) } \\
\text { involved on final } \\
\text { histology } n(\%)\end{array}$ & $\chi^{2}$ & $p$-value \\
\hline Body mass index (BMI) & & & & & 0.971 (Fisher's exact) \\
\hline Underweight & 5 & $2(3.7)$ & $3(5.3)$ & & \\
\hline Normal & 34 & $16(29.6)$ & $18(31.6)$ & & \\
\hline Overweight & 42 & $21(38.9)$ & $21(36.8)$ & & \\
\hline Obese & 30 & $15(27.8)$ & $15(26.3)$ & & \\
\hline Menopause $(n=283)$ & & & & 1.95 & 0.16 \\
\hline Premenopausal & 42 & $24(44.4)$ & $18(31.6)$ & & \\
\hline Postmenopausal & 69 & $30(55.6)$ & $39(68.4)$ & & \\
\hline NACT regimen & & & & 3.69 & 0.15 \\
\hline$A C-T$ & 95 & $43(79.6)$ & $52(91.2)$ & & \\
\hline$A C$ & 9 & $7(13)$ & $2(3.5)$ & & \\
\hline AC-TH & 7 & $4(7.4)$ & $3(5.3)$ & & \\
\hline Grade & & & & 0.66 & 0.71 \\
\hline 1 & 2 & $1(1.9)$ & $1(1.8)$ & & \\
\hline 2 & 37 & $20(37)$ & $17(29.8)$ & & \\
\hline 3 & 72 & $33(61.1)$ & $39(68.4)$ & & \\
\hline Oestrogen receptor status & & & & 7.52 & $<0.01$ \\
\hline Positive & 64 & $24(44.4)$ & $40(70.2)$ & & \\
\hline Negative & 47 & $30(55.6)$ & $17(29.8)$ & & \\
\hline Progesterone receptor status & & & & 11.27 & $<0.01$ \\
\hline Positive & 51 & $16(29.6)$ & $35(61.4)$ & & \\
\hline Negative & 60 & $38(70.4)$ & $22(38.6)$ & & \\
\hline HER2 receptor status & & & & \multicolumn{2}{|c|}{ Not analysed due to small sample size } \\
\hline Positive & 35 & $24(44.4)$ & $11(19.3)$ & & \\
\hline negative & 47 & $21(38.9)$ & $26(45.6)$ & & \\
\hline Equivocal & 22 & $6(11.1)$ & $16(28.1)$ & & \\
\hline Positive and Trastuzumab received & 7 & $3(5.6)$ & $4(7)$ & & \\
\hline Residual tumour size on final histology & & & & 24.60 & $<0.01$ \\
\hline Less than $1 \mathrm{~cm}$ & 40 & $32(59.3)$ & $8(14)$ & & \\
\hline More than $1 \mathrm{~cm}$ & 71 & $22(40.7)$ & $49(86)$ & & \\
\hline Multifocality & & & & 0.25 & 0.61 \\
\hline No & 84 & $42(77.8)$ & $42(73.7)$ & & \\
\hline Yes & 27 & $12(22.2)$ & $15(26.3)$ & & \\
\hline$N$ stage on final histology & & & & 19.80 & $<0.01$ \\
\hline 0 & 33 & $26(48.1)$ & $7(12.3)$ & & \\
\hline 1 & 29 & $13(24.1)$ & $16(28.1)$ & & \\
\hline 2 & 23 & 9 (16.7) & $14(24.6)$ & & \\
\hline 3 & 26 & $6(11.1)$ & $20(35.1)$ & & \\
\hline
\end{tabular}


In our series, women with ER positivity were less likely to have a dermal response. While $70 \%$ of ER-positive patients had dermal involvement after NACT, only $29 \%$ of ER-negative patients had dermal involvement ( $p=<0.01$ ). This is in accordance with the generally observed findings of ER-negative tumours showing better response to NACT. Ring et al [18] showed similar findings: patients with ER-negative tumours were more likely to achieve a PCR than patients who were ER-positive (21.6 versus $8.1 \%, p<0.001)$ [20].

Significant correlation was found between patients whose residual tumour size was less than $1 \mathrm{~cm}$ and no dermal involvement (Table 3 ). Out of the 40 patients with a residual size $\leq 1 \mathrm{~cm}, 32$ (80\%) patients had no dermal involvement, while out of the 71 patients who had more than $1 \mathrm{~cm}$ residual tumour involvement, 49 (69\%) patients had dermal involvement $\left(\chi^{2}=24.6, p<0.001\right)$. This is similar to findings by Shen et al [6], who in a cohort of 33 patients with T4b disease identified that up to $88 \%$ of the patients had no dermal involvement in final pathology. Cance et al [20] identified post-treatment tumour size of less than $1 \mathrm{~cm}$ as an independent prognostic factor for locoregional relapse (HR = $1.04 ; 95 \% \mathrm{Cl}=1.01-1.05, p=0.01)$.

There has been a historical bias in excluding T4b lesions from BCS for the fear of higher locoregional recurrence (LRR). However, in the few trials that have included these patients (Table 4), LRR rates have been comparable to other large breast cancers. Some studies have shown BCS to have lesser LRR than mastectomy [14]. Overall, BCS has acceptable outcomes in patient with good clinical response post-NACT in T4b lesions $[6,17,19,20]$.

Table 3. Univariate analysis of clinicopathological variables and skin (dermis) involvement.

\begin{tabular}{|c|c|c|}
\hline Variable & U-value (Mann-Whitney) & $p$-value \\
\hline Age & $8,551.500$ & 0.187 \\
\hline Time between chemotherapy and surgery & $8,917.500$ & 0.720 \\
\hline Clinical size of tumour before chemotherapy & $8,586.500$ & 0.227 \\
\hline Clinical size of tumour post-chemotherapy & $4,596.000$ & $<0.01$ \\
\hline Ratio of clinical size of tumour before chemotherapy and clinical size of tumour post-chemotherapy & $4,895.500$ & $<0.01$ \\
\hline Residual tumour size on final histology & 5,808 & $<0.01$ \\
\hline
\end{tabular}

Table 4. Comparison of data of some of the other major trials who have reported on BCS in T4b patients.

\begin{tabular}{|c|c|c|c|c|c|c|c|}
\hline Study & Inclusion criteria & $\begin{array}{c}\text { Total } \\
\text { patients }\end{array}$ & T4b patients & Tumour size & PCR & $\begin{array}{c}\text { Skin } \\
\text { involvement }\end{array}$ & $\begin{array}{c}\text { LRRat } 5 \\
\text { years in BCS }\end{array}$ \\
\hline Shen et al [6] & $\begin{array}{l}\text { T4b with good } \\
\text { response }\end{array}$ & 33 & 33 & $7 \mathrm{~cm}(2-12 \mathrm{~cm})$ & Breast 30\% & $4(12 \%)$ & $15 \%$ \\
\hline Carrara et al [17] & Stage 3 operable & 449 & 175 & $7.15+-2.15 d p$ & 77 (17.1\%) breast & - & $6 \%$ \\
\hline Mclntosh et al [14] & $\begin{array}{l}\text { Tumour greater } \\
\text { than } 4 \mathrm{~cm}\end{array}$ & 173 & 36 & - & $15 \%$ & - & $2 \%$ \\
\hline Touboul et al [19] & $\begin{array}{l}\text { Stage } 3 \text { and } \\
\text { stage } 4\end{array}$ & 137 & 34 & $6 \mathrm{~cm}(1-16 \mathrm{~cm})$ & $9 \%$ overall & - & 16 \\
\hline Cance et al [20] & $\begin{array}{l}\text { Stage } 2 \text { and } \\
\text { stage } 3\end{array}$ & 59 & 17 & - & $15 \%$ & - & $10 \%$ \\
\hline Present study & All T4b & 111 & 111 & $7 \mathrm{~cm}(2-15 \mathrm{~cm})$ & $9 \%$ overall, breast (17\%) & $51 \%$ & $\mathrm{n} / \mathrm{a}$ \\
\hline
\end{tabular}


The important question when planning BCS or SSM for T4b lesions post-NACT is whether it is safe to spare the skin post-NACT when clinical skin resolution has occurred. Our study shows that more than half of the patients achieve resolution of dermal involvement following NACT. This proportion is higher if the residual tumour is less than $1 \mathrm{~cm}$ in size or the patient is ER-negative.

When doing BCS the surgeon needs to decide whether the skin initially involved but showing clinical resolution can be spared or should be included in the surgical specimen. The authors hope the above-mentioned data will help in decision-making.

This study has many limitations. This is a retrospective study which does not allow inferring whether microscopic resolution of dermal involvement benefits in corresponding increase in disease-free survival (DFS) or overall survival (OS). This question can conclusively only be answered by fiAandomised controlled trial (RCT).

Histologically speaking, breast cancer is staged as T4b when the invasive carcinoma directly invades into the dermis or epidermis with skin ulceration. Microscopically, the tumour has to infiltrate and ulcerate the epidermis to be classified as T4b. Cases in which there is ulceration of the skin only without any infiltration of the epidermis by tumour will not be considered as T4b. Therefore, cases which grossly mimic skin involvement very often present as dermal involvement without any skin ulceration, thereby not strictly complying with the T4b criteria according to AJCC.

We have included no dermal involvement post-NACT as skin resolution (Figure 1). The AJCC criterion mentions only epidermis involvement as ypT4. If we take post-NACT AJCC criterion as skin involvement, then only 11 patients did not have skin resolution; however, that information is rather not helpful as a surgeon cannot leave behind skin where the dermis is still involved.

We have very few patients (7/42) in this subset who received anti-HER2 treatment, mainly due to financial constraints. We believe the response rates will go up if these patients were to receive Trastuzumab.

We have not classified the tumours on the present biological classification of Luminal A, B, HER2-enriched and triple negative, mainly because the HER2-enriched tumours have not been treated with targeted therapy. As targeted therapy has become more affordable in our country, we hope to provide this data in future.

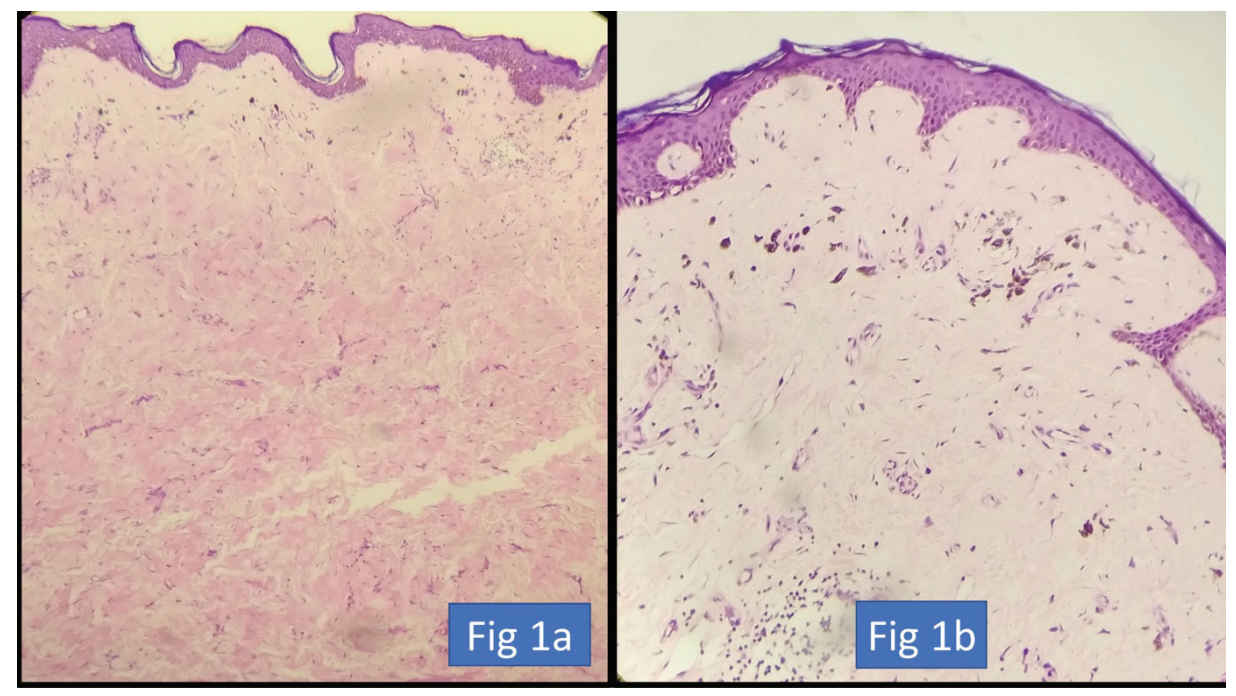

Figure 1. (a) $\mathrm{HE} \times 200$. Atrophied epidermis with loss of adnexal structures. Dermis showing hyalinisation and fibrosis indicative of treatment-related changes. (b) $\mathrm{HE} \times 400$. Dermis showing fibrosis, chronic inflammation and deposition of pigment-laden macrophages. 


\section{Conclusion}

Approximately half of T4b breast cancer shows resolution of dermal skin involvement post-NACT. ER-negative and those with residual tumour size less than $1 \mathrm{~cm}$ post-NACT are more likely to show dermal resolution. This can help surgeons plan a BCS or SSM for such patients who usually end up having a mastectomy.

\section{Abbreviations}

Neoadjuvant chemotherapy (NACT), Locally advanced breast cancer (LABC), Breast conservation surgery (BCS), Locoregional recurrence (LRR)

\section{Conflicts of interest}

The authors declare that they have no conflicts of interest.

\section{Funding}

The authors declare that no funding was received for the study.

\section{References}

1. Bray F, Ferlay J, and Seoerjomataram I, et al (2018) Global cancer statistics, 2018: GLOBOCAN estimates of incidence and mortality worldwide for 36 cancers in 185 countries CA Cancer J Clin 68(6) 394-424 https://doi.org/10.3322/caac.21492 PMID: 30207593

2. MB Amin, SB Edge, and FL Greene, et al (2017) AJCC Cancer Staging Manual 8th edn (New York: Springer)

3. Caudle AS and Kuerer HM (2014) Breast conservation therapy after neoadjuvant chemotherapy: optimization of a multimodality approach J Surg Oncol 110(1) 32-36 https://doi.org/10.1002/jso.23595 PMID: 24623334

4. Carrara GFA, Scapulatempo-Neto C, Abrahão-Machado LF, et al (2017) Breast-conserving surgery in locally advanced breast cancer submitted to neoadjuvant chemotherapy safety and effectiveness based on ipsilateral breast tumor recurrence and long-term followup Clinics (Sao Paulo) 72(3) 134-142 https://doi.org/10.6061/clinics/2017(03)02

5. Agarwal G and Ramakant P (2008) Breast cancer care in India: current situation and challenges for the future Breast Care (Basel) 3 21-27 https://doi.org/10.1159/000115288

6. Shen J, Valero V, and Buchholz TA, et al (2004) Effective local control and long-term survival in patients with T4 locally advanced breast cancer treated with breast conservation therapy Ann Surg Oncol 11(9) 854-860 https://doi.org/10.1245/ASO.2004.02.003 PMID: 15313733

7. American Joint Committee for Cancer Staging and End Results Reporting (1977) Manual for Staging of Cancer (Chicago: American Joint Committee)

8. Singletary SE (2001) Neoadjuvant chemotherapy in the treatment of stage II and III breast cancer Am J Surg 182 $341-346$ https://doi. org/10.1016/S0002-9610(01)00724-3 PMID: 11720667 
9. Kuerer HM, Hunt KK, and Newman LA, et al (2000) Neoadjuvant chemotherapy in women with invasive breast carcinoma: conceptual basis and fundamental surgical issues J Am Coll Surg 190 350-363 https://doi.org/10.1016/S1072-7515(99)00272-0 PMID: 10703863

10. Guth U, Wight E, and Schotzau A, et al (2005) Breast carcinoma with noninflammatory skin involvement (T4b): time to abandon an historic relic from the TNM classification Cancer 104(9) 1862-1870 https://doi.org/10.1002/cncr.21394 PMID: 16130140

11. Silverman D, Ruth K, and Sigurdson ER, et al (2014) Skin involvement and breast cancer: are T4b lesions of all sizes created equal? J Am Coll Surg 219(3) 534-544 https://doi.org/10.1016/j.jamcollsurg.2014.04.003 PMID: 25026875 PMCID: 4143438

12. Calais G, Berger C, and Descamps P, et al (1994) Conservative treatment feasibility with induction chemotherapy, surgery, and radiotherapy for patients with breast carcinoma larger than $3 \mathrm{~cm}$ Cancer 74 1283-1288 https://doi.org/10.1002/10970142(19940815)74:4<1283::AID-CNCR2820740417>3.0.CO;2-S PMID: 8055449

13. Scholl SM, Fourquet A, and Asselain B, et al (1994) Neoadjuvant versus adjuvant chemotherapy in premenopausal patients with tumours considered too large for breast conserving surgery: preliminary results of a randomized trial: S6 Eur J Cancer 30A 645-652 https://doi. org/10.1016/0959-8049(94)90537-1

14. McIntosh SA, Ogston KN, and Payne S, et al (2003) Local recurrence in patients with large and locally advanced breast cancer treated with primary chemotherapy Am J Surg 185 525-531 https://doi.org/10.1016/S0002-9610(03)00078-3 PMID: 12781879

15. Guth U, Singer G, and Schötzau A, et al (2005) Scope and significance of non-uniform classification practices in breast cancer with non-inflammatory skin involvement: a clinicopathologic study and an international survey Ann Oncol 16 1618-1623 https://doi. org/10.1093/annonc/mdi319 PMID: 16033873

16. Wittekind C, Henson DE, and Hutter RVP, et al (2001) TNM supplement A Commentary on Uniform Use 2nd edn, eds P Hermanek, LH Sobin, and RVP Hutter, et al (New York: Wiley-Liss)

17. Carrara GF, Scapulatempo-Neto C, and Abrahão-Machado LF, et al (2017) Breast-conserving surgery in locally advanced breast cancer submitted to neoadjuvant chemotherapy safety and effectiveness based on ipsilateral breast tumor recurrence and long-term followup Clinics (Sao Paulo) 72(3) 134-142 https://doi.org/10.6061/clinics/2017(03)02

18. Ring A, Smith I, and Ashley S, et al (2004) Oestrogen receptor status, pathological complete response and prognosis in patients receiving neoadjuvant chemotherapy for early breast cancer Br J Cancer 91 2012-2017 https://doi.org/10.1038/sj.bjc.6602235 PMID: 15558072 PMCID: 2409783

19. Touboul E, Lefranc JP, and Blondon J, et al (1997) Primary chemotherapy and preoperative irradiation for patients with stage II larger than $3 \mathrm{~cm}$ or locally advanced non-inflammatory breast cancer Radiother Oncol 42 219-229 https://doi.org/10.1016/S01678140(97)01923-3 PMID: 9155070

20. Cance WG, Carey LA, and Calvo BF, et al (2002) Long-term outcome of neoadjuvant therapy for locally advanced breast carcinoma: effective clinical downstaging allows breast preservation and predicts outstanding local control and survival Ann Surg 236 295-303 https://doi.org/10.1097/00000658-200209000-00006 PMID: 12192316 PMCID: 1422583 